\title{
Le sens du Nord dans l'éducation et la formation au lieu : Exploration d'enjeux
}

Carlo Prévil et Katerin Arias-Ortega

\section{OpenEdition}

1 Journals

Édition électronique

URL : https://journals.openedition.org/ere/7813

DOI : 10.4000/ere.7813

ISSN : 2561-2271

Éditeur

Centr'ERE

Référence électronique

Carlo Prévil et Katerin Arias-Ortega, « Le sens du Nord dans l'éducation et la formation au lieu

Exploration d'enjeux», Éducation relative à l'environnement [En ligne], Volume 16-2 | 2021, mis en ligne le 14 octobre 2021, consulté le 09 février 2022. URL : http://journals.openedition.org/ere/7813; DOI : https://doi.org/10.4000/ere.7813

Ce document a été généré automatiquement le 9 février 2022

La revue Éducation relative à l'environnement est mise à disposition selon les termes de la Licence Creative Commons Attribution - Pas d'Utilisation Commerciale 4.0 International. 


\title{
Le sens du Nord dans l'éducation et la formation au lieu : Exploration d'enjeux $^{1}$
}

\author{
Carlo Prévil et Katerin Arias-Ortega
}

L'éducation et la formation au monde social (Audigier, 2012) visent à former des apprenants cultivés et à les doter d'une vision du territoire comme espace aménagé et approprié par les humains, et auquel ils se sont adaptés (Québec, 2010). Bone (2016) considère le Canada comme "un pays de régions ", dont le Nord est la plus difficile à définir et à caractériser en raison de sa diversité géographique, sociale et culturelle. En effet, avec plus de quatre millions de $\mathrm{km}^{2}$, le Nord constitue environ $40 \%$ de l'ensemble du Canada (Hamelin, 1976). Il s'étend au-delà du $49^{\mathrm{e}}$ parallèle au Québec et du $55^{\mathrm{e}}$ pour le reste du Canada. À partir du $60^{\mathrm{ème}}$ parallèle, on parle du Grand Nord (Figure 1). 


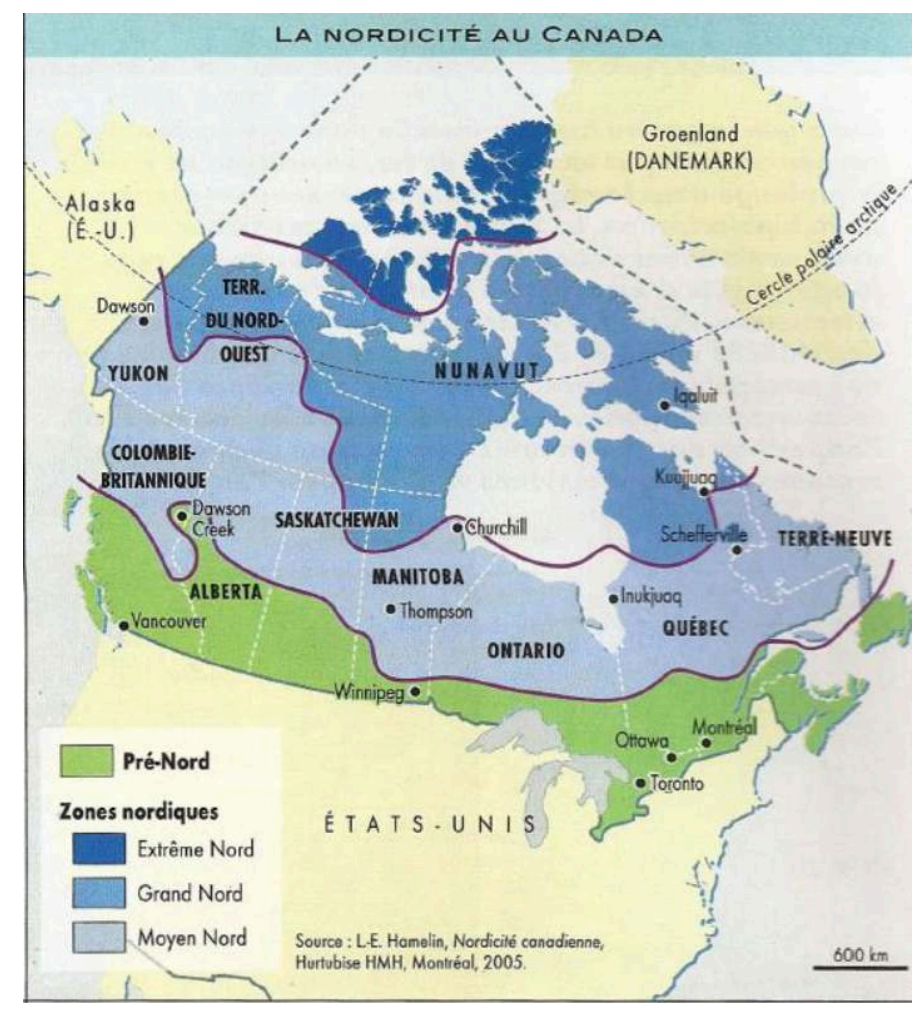

Figure 1 : Nordicité au Canada (Bayly, 2018)

Le Nord du Canada évolue entre des enjeux géopolitiques, avec plus de 25 pays au Conseil de l'Arctique ${ }^{2}$, et des enjeux territoriaux internes en raison de ses défis sociaux, économiques et culturels. Il forme une triade : « celle du climat froid, de la population clairsemée et du peuplement où dominent les Amérindiens et les Inuit » (Rougier, 1991, p. 223). Mitcham (1983) évoque le Nord du Canada comme la dernière frontière de l'Amérique, alors qu'Hulan (2014) retrace de manière contrastée les tensions entre un Nord vécu, un Nord imaginaire et un Nord idéologique dans l'esquisse d'une identité nationale canadienne.

Les différents systèmes éducatifs provinciaux identifient un ensemble de savoirs, de savoir-faire et d'attitudes servant à développer chez les apprenants des compétences fondamentales, instrumentales et transversales (Peters, 2015). Le Programme de formation de l'école québécoise (PFEQ) désigne l'histoire, la géographie et l'éducation à la citoyenneté comme les disciplines prépondérantes pour étudier l'univers social (Québec, 2010). Du même coup, l'éducation géographique y est considérée comme le fondement du domaine d'apprentissage du monde social (Audigier, 2015 ; Québec, 2010) pour favoriser chez les apprenants le développement de compétences relatives à l'environnement, la consommation, la diversité, le vivre-ensemble et la citoyenneté (Québec, 2010). Dans ce contexte, quelle éducation au sens du Nord faut-il offrir au Québec et au Canada pour répondre aux défis du vivre-ensemble, tout en formant des individus épanouis, de futurs citoyens engagés et des travailleurs qualifiés?

Dans cet article, nous explorons les questionnements ontologiques propices au développement d'une éducation au sens $d u$ Nord et capables d'en intégrer les complexités culturelles et socioécologiques (Steele, 1981 ; Tuan, 2001). Nous explicitons des repères théoriques associés aux enjeux émergents à l'interface du développement 
socioterritorial, de l'environnement et de l'autochtonie du Nord. Ensuite, nous définissons un cadre conceptuel permettant l'interconnexion de l'éducation, de la géographie et de la culture comme piliers fondamentaux de l'éducation au sens $d u$ Nord. Finalement, nous passons en revue des approches disponibles pour la mise en pratique de l'éducation au sens $d u$ Nord comme nouvel objet d'apprentissage à promouvoir au Québec et au Canada (Cannatella, 2007 ; Girard et Brisson, 2018 ; Kudryavtsev et coll., 2012 ; Mérenne-Schoumaker, 2017). Cette réflexion vise à contribuer aux débats sur le renouvellement de l'éducation géographique pour mieux comprendre le sens du Nord, le promouvoir comme nouvel objet d'apprentissage et enrichir les finalités des programmes de formation (Asselin, 2011 ; Hamelin, 1976).

\section{Éducation géographique et éducation au sens $d u$ Nord}

Pélissier (1989) rappelle qu'« [...] il faut enseigner la géographie parce qu'elle restitue à chaque société (et à chaque citoyen), à partir d'un diagnostic de situation, sa mémoire et ses racines »(p.185). En ce sens, l'éducation géographique s'inscrit dans une démarche de transmission culturelle relativement à l'humanisation de la terre (Colin, 2013 ; Merenne-Schoumaker, 2017). La culture géographique s'établit à travers des concepts fondamentaux (lieu, espace, paysage, région, territoire), structurants (localisation, association, organisation) et sous-jacents aux processus géographiques (dynamique spatiale, interaction, flux) (Benimmas et Blain, 2019). Ainsi, la géographie caractérise les lieux selon différentes perspectives: lieux de vie, lieux de pouvoir et lieux de dynamiques spatiales d'humanisation (Colin, 2013). Les lieux de vie intersectent des relations verticales conditionnant le fonctionnement des habitats, qu'ils soient éphémères ou persistants (campement, milieu urbain, iglou ou parcours de chasse) (Pumain et Saint-Julien, 1997). Les lieux de pouvoir se situent au cœur des processus décisionnels en vue de l'appropriation et de l'exploitation des ressources du milieu, ou pour débattre sur des enjeux territoriaux selon les contraintes et les atouts répertoriés comme des marqueurs de relations horizontales des territoires. Or, de tels marqueurs sont valorisés selon l'identité, la culture et les représentations que se font les acteurs de ces lieux de pouvoir (territoires ancestraux, région minière, aménagement urbain) (Anderson, 2006 ; Audigier, 2015 ; Scheibling, 2011). Finalement, les lieux de dynamiques spatiales d'humanisation s'inscrivent dans des relations temporelles à travers des transitions, des interactions ou des flux. Ces dynamiques modulent les formes de proximité, les relations horizontales, les relations verticales et l'enchainement des processus en cours entre les unités spatiales (rivage de rassemblement du clan, barrage hydro-électrique ou reprofilage paysager par exemple) (Niens et Reilly, 2012).

Dans le PFEQ, l'éducation géographique au primaire aborde l'éducation au Nord à travers une seule thématique au $3^{\mathrm{e}}$ cycle (10-12 ans) (Déry, 2016 ; Québec, 2010). En lien avec le développement de la compétence sur la diversité du monde social sur le territoire, cette thématique porte sur les Inuits comme peuple du Nord, Au secondaire, en géographie ( $1^{\mathrm{re}}$ et $2^{\mathrm{e}}$ années, $12-15$ ans), le territoire-région est étudié dans le contexte de la compétence sur l'interprétation d'un enjeu territorial. En privilégiant l'angle de l'enjeu environnemental, le PFEQ circonscrit le Nord à la production énergétique et valorise une vision développementiste. Finalement, le Nord est étudié accessoirement comme territoire-autochtone relativement aux revendications 
territoriales et aux traités (Hamelin, 2006; Québec, 2010). Une telle approche évoque davantage des tensions et des ressentiments, sans considérer la complexité des nombreux enjeux, au nord comme au sud, associés à l'identité, au mode de vie et au développement socioterritorial ${ }^{3}$ (Québec, 2010). Dans tous les autres cycles de formation, incluant l'université, il n'existe aucun référentiel transversal pour la formation et l'acquisition de compétences et de savoirs intégrés relatifs aux territoires et aux sociétés du Nord (Bouchard et Désy, 2013 ; Desbiens, 2012 ; Hamelin, 2006). Le peu de visibilité accordé aux réalités du Nord est de nature à entretenir des perceptions négatives (froideur, isolement, précarité, revendications pressantes des Autochtones), et à entraver le développement d'une identité culturelle partagée et d'un sentiment commun d'appartenance territoriale (Audigier, 2012). Cette éducation géographique lacunaire sur le sens $d u$ Nord limite la formation des nouvelles générations sur de nombreuses questions vives portant sur l'environnement et la consommation, la diversité, le vivre-ensemble et la citoyenneté.

Le Nord fait l'objet d'une attention soutenue de la part du gouvernement du Canada depuis le tournant du millénaire. L'ancien premier ministre Stephen Harper (2006-2015) déclarait à l'occasion du lancement de la Stratégie pour le Nord du Canada: "Le Canada est un pays nordique. Le vrai Nord est l'avenir des explorateurs, des entrepreneurs et des artistes canadiens. Nous tournerions le dos à notre identité en tant que Canadiens si nous n'agissions pas sur le potentiel de cette région, le vrai Nord, qui entreprend actuellement son ascension. » (Canada, 2009). Ce regain d'intérêt pour le Nord est attesté dans un premier sommaire de faits marquants des strates décisionnelles fédérales compilés dans le Tableau 1.

Tableau 1 : Le Canada et le Nord

\begin{tabular}{|c|c|c|}
\hline Année & Entité & Initiative \\
\hline 2001 & $\begin{array}{l}\text { Forum des } \\
\text { Ministres }\end{array}$ & $\begin{array}{l}\text { Désignation de responsables du } \\
\text { développement du Nord }\end{array}$ \\
\hline 2002 & $\begin{array}{l}\text { Centre de } \\
\text { recherche sur les } \\
\text { sciences naturelles } \\
\text { et génie (CRSNG) }\end{array}$ & $\begin{array}{l}\text { Création de six (6) Chaires de recherche } \\
\text { sur le Nord dans les universités au Canada }\end{array}$ \\
\hline 2003 & $\begin{array}{l}\text { Min. des Affaires } \\
\text { indiennes et du } \\
\text { Nord canadien }\end{array}$ & $\begin{array}{l}\text { Publication des Lignes directrices sur } \\
\text { l'aménagement des terres du Nord }\end{array}$ \\
\hline $\begin{array}{l}2007- \\
2008\end{array}$ & $\begin{array}{l}\text { Année Polaire } \\
\text { Internationale }\end{array}$ & $\begin{array}{l}\text { Inauguration du brise-glace Amundsen. } \\
\text { Lancement du Réseau de Centres } \\
\text { d'excellence Arctic Net ( } 100 \text { chercheurs } \\
\text { et } 27 \text { universités au Canada) }\end{array}$ \\
\hline 2008 & Canada & $\begin{array}{l}\text { Mise en place de la Stratégie pour le Nord } \\
\text { du Canada }\end{array}$ \\
\hline 2011 & Canada & $\begin{array}{l}\text { Création du ministère des Affaires } \\
\text { Autochtones et Développement du Nord }\end{array}$ \\
\hline 2017 & Canada & $\begin{array}{l}\text { Création des ministères: Relations } \\
\text { Couronne-Autochtones et Affaires du } \\
\text { Nord et Services aux Autochtones }\end{array}$ \\
\hline 2018 & & $\begin{array}{l}\text { Transfert des Affaires du Nord au } \\
\text { nouveau ministère des Affaires } \\
\text { intergouvernementales et du Nord et du } \\
\text { Commerce intérieur }\end{array}$ \\
\hline 2020 & Canada & $\begin{array}{l}\text { Publication du Cadre stratégique pour } \\
\text { l'Arctique et le Nord du Canada }\end{array}$ \\
\hline
\end{tabular}


8 Ce même intérêt pour le Nord ${ }^{4}$ peut être retracé dans l'établissement de différents instruments d'aménagement du territoire au sein de plusieurs provinces et territoires du Canada (Tableau 2).

Tableau 2 : Gouvernances territoriales au Nord du Canada

\begin{tabular}{|c|c|c|}
\hline Année & $\begin{array}{c}\text { Entité } \\
\text { territoriale }\end{array}$ & Initiative \\
\hline 1998 & Saskatchewan & $\begin{array}{l}\text { Northern Saskatchewan's Planning for } \\
\text { Growth program }\end{array}$ \\
\hline 1999 & Nunavut & Création du Territoire fédéral du Nord \\
\hline 2000 & Manitoba & $\begin{array}{l}\text { Large Area Land-Use Planning } \\
\text { (LALUP) }\end{array}$ \\
\hline 2004 & $\begin{array}{l}\text { Colombie- } \\
\text { Britannique }\end{array}$ & Northern Development Initiative Trust \\
\hline 2005 & Nunatsiavut & $\begin{array}{l}\text { Création du territoire autonome géré } \\
\text { par les Inuits de Terre-Neuve-et- } \\
\text { Labrador }\end{array}$ \\
\hline 2007 & $\begin{array}{l}\text { Terre-Neuve-et- } \\
\text { Labrador }\end{array}$ & Northern Strategic Plan for Labrador \\
\hline 2008 & Alberta & Province's Land Use Framework \\
\hline 2010 & Ontario & Growth Plan for Northern Ontario \\
\hline 2011 & Québec & Le Plan Nord \\
\hline
\end{tabular}

9 Au Québec, plusieurs initiatives des gouvernements et de la société civile depuis le tournant du siècle méritent également d'être répertoriées (Tableau 3). De même, de nombreux débats sociaux ont traité de cette question ${ }^{5}$.

Tableau 3 : Le Québec et le Nord

\begin{tabular}{llc}
\hline Année & \multicolumn{1}{c}{ Instance } & Initiative \\
\hline 2010 & $\begin{array}{l}\text { Plan Nord (Parti Libéral } \\
\text { du Québec) }\end{array}$ & 85 milliards \$ / 30 ans \\
\hline 2013 & $\begin{array}{l}\text { Plan Nord 2.0 (Parti } \\
\text { Québécois) }\end{array}$ & 20 milliards \$ / 10 ans \\
\hline 2014 & $\begin{array}{l}\text { Nouveau Plan Nord (Parti } \\
\text { Libéral du Québec) }\end{array}$ & 50 milliards \$ / 20 ans \\
2019 & $\begin{array}{l}\text { Nord pour Tous (Coalition } \\
\text { Avenir Québec) }\end{array}$ & 30 milliards \$ / 12 ans \\
\hline
\end{tabular}

Dans ces conditions, il devient important de définir et de conceptualiser les savoirs essentiels à enseigner, de même que les valeurs et les attitudes à transmettre par les 
passeurs culturels en éducation au sens $d u$ Nord. Ces savoirs peuvent varier en complexité selon les identités des parties prenantes ou selon ce qu'un acteur social aura respectivement vu, perçu, imaginé, vécu ou représenté relativement au Nord (Grace, 2007 ; Hulan, 2014 ; Pichon, 2015). L'importance de mieux conceptualiser le sens du Nord comme objet d'apprentissage s'impose pour former les apprenants à interpréter les enjeux multiples des lieux associés au Nord (Cheng et coll., 2003 ; Scannell et Gifford, 2010).

\section{Dans le Nord du Québec et du Canada : Affirmation d'une autochtonie}

Le Nord du Canada est souvent caractérisé par son immensité, ses ressources naturelles et ses températures froides. Il est également considéré comme atypique, vu son peuplement humain dominé par les Premières nations et les Inuits (Hamelin, 2006; Mowat, 2009; Rougier, 1991). Le nord du Québec couvre plus de $70 \%$ du territoire provincial pour moins de $10 \%$ de la population (Stat-Can, 2017). Environ $30 \%$ de cette population se réclame de l'identité autochtone, alors que celle-ci est revendiquée par moins de $2 \%$ de la population pour le reste du territoire (StatCan, 2017). Les populations autochtones du Nord, longtemps négligées par le reste du pays, ont maintenu la permanence de l'occupation de l'espace avant un certain regain d'intérêt des communautés du Sud, notamment à partir des années 1950 pour des approches volontaristes d'aménagement de ce vaste territoire (Bocking et Martin 2017 ; CRV, 2015 ; Nungak, 2019). Les activités principales des communautés autochtones du Nord demeurent la chasse, la pêche, le piégeage et l'artisanat, même si ces pratiques intègrent aujourd'hui les technologies du Sud (motoneige, aviation, habitat permanent) à côté de nouveaux créneaux d'activités économiques (de l'exploitation minière et pétrolière jusqu'au tourisme) (Bayly, 2018). Dans les Plans Nord (Tableau 3), les potentialités du territoire sont définies essentiellement selon une vision extractiviste des ressources. Les retombées potentielles s'évaluent dans une vision d'économie mondialisée bien qu'elles puissent être inégales au sein des différentes communautés impliquées (communautés autochtones, entreprises multinationales, travailleurs qualifiés venus du Sud, etc.) et d'une faible acceptabilité sociale (Asselin, 2011; Bouchard et Désy, 2013 ; Nungak, 2019).

12 Au Nord, les Autochtones se considèrent comme des habitants permanents avec une économie basée sur les ressources renouvelables (Nungak, 2019). Ils réclament une plus grande implication dans le développement du territoire en vue de la création d'une économie autochtone et d'institutions politiques reconnaissant leur présence et leurs revendications territoriales (Bayly, 2018; Bouchard et Désy, 2013) : «ils veulent corriger les types de sous-développement et de relations Nord-Sud dont ils sont l'objet» (Rougier, 1991, p.225). Cette reconnaissance serait essentielle pour la protection de leur identité (langues, art, cultures, valeurs), de leur économie (ressources renouvelables) et pour leur participation à l'économie des ressources non renouvelables (Feit, 2018). Elle permettrait de résoudre les fractures entre communautés autochtones et non-autochtones sur les enjeux liés au sens du Nord (Anderson, 2006 ; Bocking et Martin, 2017 ; Bouchard et Désy, 2013 ; Girault et Barthes, 2016). 
13 Dans les communautés autochtones, les territoires sont perçus comme ancestraux et dépourvus d'un statut de propriété défini par le Code civil (Québec) ou la Common Law (Canada) (Hamelin, 1976 ; Scott, 2013). Hamelin (2006) souligne que les enjeux du Nord réactualisent le principe de l'Until Policy ${ }^{6}$ pour l'aménagement du territoire et nécessitent une nouvelle éducation sur le sens du lieu. Le principe de l'Until Policy, de la clause autochtone de la Constitution de 1982, renferme des dispositions pour promouvoir la réconciliation et améliorer les relations entre les communautés autochtones et non-autochtones sur le sens du Nord (Barnhardt, 2008; Battiste, 2013 ; CRV, 2015).

14 Les territoires du Nord sont actuellement en proie à de nombreuses transformations. Ainsi, trois grands défis de société justifient la reconnaissance de l'urgence de l'éducation au sens $d u$ Nord comme un enjeu fondamental du domaine d'apprentissage du monde social: a) l'évolution des changements climatiques et la nécessité de la recherche de scénarios d'adaptation de proximité pour les milieux très fragiles comme le Nord (Ouranos, 2015) ; b) les effets de la mondialisation et la demande par les pays développés et émergents de matières premières dont regorgerait le Nord (Canada, 2009; Québec, 2010); c) la dynamique socio-territoriale et culturelle singulière du Canada, marquant un ancrage identitaire et culturel prédominant des Premiers Peuples dans le Nord (Canada, 2009 ; Hamelin, 1976 ; Mowat, 2009).

Cette éducation au sens du Nord est de nature à renforcer les finalités du domaine d'apprentissage du monde social ${ }^{7}$ et à contribuer au développement d'une compétence transversale au sens du lieu, propice à l'écocitoyenneté, c'est-à-dire la nécessité, pour l'individu, d'avoir des gestes et des comportements responsables envers son lieu de vie et ses semblables. Sauvé (2014) envisage cette écocitoyenneté comme « une citoyenneté critique, compétente, créative et engagée, capable et désireuse de participer aux débats publics, à la recherche de solutions et à l'innovation écosociale» (p. 21). En effet, la nécessité de mieux intégrer les communautés autochtones avec leurs valeurs et leurs cultures dans les débats portant sur les défis de l'environnement et le développement socioterritorial renforce l'importance de savoirs essentiels partagés en éducation sur le sens du lieu et particulièrement sur le sens du Nord, en reconnaissance de l'autochtonie de cette région et pour contribuer à la décolonisation de l'éducation (Bocking et Martin 2017 ; Chartier, 2014 ; Hamelin, 1976). L'autochtonie, sens de l'identité territoriale et culturelle, s'inscrit dans des schémas de perceptions et de conceptions qui émergent à travers une construction particulière de l'éducation géographique et du sens du lieu, et ce, jusqu'à l'engagement pour l'écocitoyenneté. Elle renouvelle les projets territoriaux dans l'idée que "[...] cette symbiose entre l'espace et la société est aussi de nature spirituelle. Le pays et le peuple ne font qu'un, et là est la clé de la survivance : [puisque] land is not money, land is life [...]» (Rougier, 1991, p. 232).

16 L'éducation au sens du Nord en géographie peut engendrer une transformation considérable des contenus enseignés tout au long de la vie et la nature des dispositifs et des méthodes d'enseignement (Audiger, 2012; Mérenne-Schoumaker, 2019). Elle s'avère essentielle pour les différentes catégories d'apprenants: élèves, futurs enseignants, enseignants en exercice et tout autres apprenants, pour sa formation sociale générale, ce qui entraine la nécessité d'une mise en perspective des interconnexions de la géographie, de la culture et de l'éducation pour appréhender de nouvelles dynamiques spatiales d'humanisation au sens du Nord (Audiger, 2012 ; Prévil et Arias-Ortega, 2020). 


\section{Interconnexions de l'éducation, de la géographie et de la culture}

17 Mialaret (2006) définit l'éducation comme un processus de transmission culturelle et sociale par lequel une génération donnée lègue à la suivante des savoirs, des valeurs et des attitudes visant à la fois la socialisation des nouveaux membres et l'actualisation optimale de toutes leurs potentialités. Dans cette perspective, l'éducation géographique permet aux apprenants d'accéder à des connaissances sur les territoires par le prisme : a) des savoirs issus de l'observation directe et indirecte de phénomènes socioterritoriaux; b) des savoirs à modéliser dans les systèmes d'information géographique ; c) des savoirs à représenter pour communiquer, discuter et débattre des enjeux territoriaux ou des scénarios d'aménagement. L'éducation géographique doit répondre à la demande sociale d'éducation et de formation de citoyens capables de partager des points de vue multiples sur le territoire (Audigier, 1997 et 2012 ; Prévil, 2009). Elle doit former l'esprit critique et intégrer des aptitudes pour appréhender et comprendre d'une part, ce qui est commun et partagé par l'ensemble de la société, et qui vise l'harmonisation des relations sociales (vivre-ensemble et citoyenneté) (Bouchard et Désy, 2013; Chartier 2014; Québec, 2010) et d'autre part, ce qui est spécifique selon les contextes, les situations, les cultures (autochtones ou nonautochtones, par exemple) et découle de constructions et de traitements originaux (diversité, environnement et consommation) (Audigier, 2012 ; Canobbio, 2009 ; Québec, 2010). La culture se conçoit comme un système d'éléments matériels, humains et spirituels, caractérisé par un certain équilibre permettant aux individus d'un groupe particulier de satisfaire des besoins fondamentaux grâce à des compétences particulières (Semin, 2009). Il s'établit ainsi une relation entre ces individus pour le partage des connaissances et des savoirs accumulés par le groupe dans un environnement naturel ou social (Arendt, 1972). Cette construction permet la mise en forme et l'organisation des croyances, du comportement et des systèmes de valeurs comme une géopoétique (Déry, 2016 ; Semin, 2009).

18 L'interconnexion « éducation-géographie-culture » permet les apprentissages suivants : 1) des valeurs, croyances, comportements et symboles de la socialisation partagés par un groupe particulier ; 2) des comportements humains escomptés en fonction de situations ou de lieux spécifiques; 3) des savoirs-agir dans certains lieux et dans certaines situations données, et découlant de l'expérience sociale passée ; 4) des objets matériels, humains et spirituels grâce auxquels l'être humain est capable d'affronter les problèmes du quotidien à partir d'un cadre de référence partagé (Cossio, 2015).

19 Les approches épistémologiques associées à la culture géographique sont reliées à des valeurs à considérer pour l'éducation géographique (Bonnemaison, 2000 ; Claval, 2012 ; Colin, 2013). Elles aident à saisir l'importance de l'information dans les processus de coconstruction de savoirs et de géosymboles du territoire (Bonnemaison, 2000) comme espace informé, par et pour les acteurs territoriaux (François, 2008), rendant les lieux davantage significatifs grâce à des relations entre les imaginaires géographiques et les représentations (Anderson, 2006; Claval, 2012; Hulan, 2014; Pichon, 2015). Elles favorisent aussi la mutualisation des référentiels de formation des apprenants sous l'angle des identités territoriales et culturelles (Claval 2012; Girault et Barthes, 2016), et la négociation des décisions territoriales à travers un sens du Nord partagé (Hulan, 
2014 ; Mérenne-Schoumaker, 2017). Dans la structuration complexe de cette relation de la société avec l'espace surgit une double ouverture pour la conceptualisation du sens du lieu sSense of place). Il s'agit d'un côté de contribuer à la définition des identités territoriales et culturelles Nord/Sud (place attachment) en s'appuyant sur les lieux de vie et les lieux de pouvoir (Steele, 1981; Tuan, 2001) et, de l'autre, de pouvoir mieux négocier au sein de la gouvernance territoriale Sud/Nord (place meaning) en s'appuyant sur les lieux de pouvoir et les lieux de dynamiques spatiales d'humanisation (Canobbio, 2009 ; Girault et Barthes, 2016 ; Steele, 1981 ; Tuan, 2001).

20 À la suite d'Arendt (1972), Audigier (1997) a identifié six types de finalités du système éducatif, à savoir des finalités intellectuelles, professionnelles, pratiques, patrimoniales, culturelles et critiques. Les savoirs produits et vulgarisés sur le Nord sont ceux nécessaires à la gouvernance des ressources territoriales du Nord et à la formation de travailleurs compétents dans une économie mondialisée, ce qui correspond aux trois premières finalités (Québec, 2010; Barnhart, 2013; Griffiths et coll., 2015). Les trois autres restent non avenues. Il apparait ainsi une double fracture Nord/Sud sur la transmission des savoirs territoriaux, des valeurs et des attitudes, en vue de la formation d'individus épanouis et de citoyens engagés en accord avec des finalités patrimoniales, culturelles et critiques (Audigier, 1997 ; Mérenne-Schoumaker, 2017).

21 Une éducation au sens $d u$ Nord permettra de redécouvrir et d'intégrer le Nord comme lieu de vie, de mieux problématiser le Nord comme lieu de pouvoir et de coopérer dans les rapports sociaux au Nord relativement à l'habitat, aux transactions et à la décision territoriale (Chartier, 2014 ; Prévil, 2009 ; Québec, 2010; Sauvé et coll., 2017). Elle sera essentielle pour participer aux débats socio-territoriaux portant sur le Nord comme questions socialement vives en raison des enjeux de l'environnement, du développement socioterritorial et de l'autochtonie du Nord (Audigier, 1997 et 2012; Bouchard et Désy, 2013). Les compétences associées à ce nouvel objet d'apprentissage viendront appuyer la construction d'identités et de valeurs nécessaires au renforcement de l'écocitoyenneté et inspirer la gouvernance équilibrée des transformations socioterritoriales, au Nord comme au Sud (Cheng, Kruger et Daniels, 2003 ; Lengen et Kistemann, 2012). L'éducation géographique au sens $d u$ Nord s'insère dans un champ conceptuel à l'interface a) du lieu (Place) (Mérenne-Schoumaker, 2019), b) de l'interculturalité, pour tenir compte des intersections identités/territoire et Autochtone/non-Autochtone (Anderson, 2006 ; Bouchard et Désy, 2013 ; Campeau 2009) et c) de l'écocitoyenneté pour les apprentissages collectifs nécessaires à l'intégration des défis de l'adaptation aux changements socioterritoriaux (Niens et Reilly, 2012; Sauvé et coll., 2017). Il convient maintenant d'exposer quelques grandes lignes pouvant être nécessaires à la mise en œuvre de telles compétences.

\section{Approches permettant de contextualiser le sens $d u$ Nord comme objet d'apprentissage}

Partant des enjeux de l'éducation au sens $d u$ Nord discutés ci-dessus, nous présentons six propositions éducatives pouvant contribuer à contextualiser le sens du Nord: la nordicité, la géopoétique nordique, le développement durable, le cadre d'Éducation 2030, l'éducation environnementale post-Rio et l'éducation et la pédagogie autochtones. Ces propositions peuvent contribuer chacune à intégrer les défis de 
l'environnement, du développement socioterritorial et de l'autochtonie du Nord. De cette intégration pourront surgir les axes et les dimensions d'une éducation géographique renouvelée capable d'articuler les complexités du sens $d u$ Nord comme nouvel objet d'apprentissage au Québec et au Canada.

\section{Nordicité}

La nordicité se définit comme «la polaricité de l'hémisphère boréal » (Hamelin, 1976). Elle intègre « un questionnement de sens, d'état, d'identité et d'objet au sujet de la zone froide de l'hémisphère boréal » (Hamelin, 1976, p.3). La nordicité géographique se calcule et s'exprime en valeurs polaires (VAPO), attribuées selon dix indicateurs allant de l'identification de la latitude jusqu'aux activités économiques (Hamelin, 2006). En associant des imaginaires, des données et des représentations, ces indicateurs permettent d'établir des lignes d'isonord (d'égales valeurs nordiques) contribuant à zoner de manière comparative les caractéristiques territoriales et régionales de l'ensemble du Québec (Hamelin, 1976). Desbiens (2012) souligne ainsi que la nordicité désigne " un processus d'échange interculturel qui, à son tour, peut soutenir une identification territoriale plurielle dans un Québec qui serait à la fois solidaire et diversifié » (p. 656), un ensemble d'idées pouvant contribuer à l'éducation au sens du Nord comme lieu de vie et lieu de pouvoir.

\section{Géopoétique nordique}

La géolittératie et la géopoétique associent la toponymie et la connaissance des lieux aux autres sources d'expression du ressenti, des imaginaires, des savoirs locaux et des valeurs populaires. Elles constituent une perspective originale pour l'éducation et la formation au Nord (Canobbio, 2009 ; Cottereau, 1999). À travers la géopoétique, on retrouve la mesure du ressenti et l'expression de l'attachement dans la modulation du vécu et du perçu (Cheng et al, 2003 ; Vaillancourt 2017). La littérature, la sculpture, les chants, les danses, la poésie, les contes ou la musique issus des gens du Nord peuvent intégrer une géopoétique pour raconter les lieux, le territoire et le monde social d'une manière authentique (Bouvet, 2015). Cette conceptualisation aiderait à porter l'éducation au sens $d u$ Nord dans des horizons nouveaux pour l'intégration et la caractérisation des savoirs à apprendre sur le sens $d u$ Nord, particulièrement comme lieu de pouvoir (Déry, 2016 ; Vaillancourt, 2017).

\section{Éducation au développement durable}

Les projets de développement économique dans le Nord pour l'ensemble du Canada évoquent à chaque fois les échos des revendications des groupes sociaux pour introduire l'idée d'un développement durable du Nord. Le Plan Nord, présenté en 2012 au Québec, s'est réclamé du " développement durable » tel que défini par les Nations Unies en 1992. En 2006, l'Assemblée nationale du Québec a adopté à l'unanimité la Loi sur le développement durable (Québec, 2006). Depuis, les différentes versions du Plan Nord ont toujours été assujetties à cette loi, ainsi que les principes et indicateurs afférents (Documents du Plan Nord, 2019). Ces indicateurs se rapportent à la biodiversité, au climat, au sentiment d'appartenance et au développement culturel (Québec, 2012). De même, les principes de participation du public, de précaution, de protection du 
patrimoine culturel, de respect de la capacité de soutien des écosystèmes permettent de se rapprocher de la nécessité d'intégrer autant les faits que les imaginaires (Québec, 2012). Ce discours suggère des pistes pour l'éducation et la formation au sens $d u$ Nord principalement comme lieu de dynamiques spatiales d'humanisation.

\section{Cadre d'action Éducation 2030} la culture, de l'éducation et de l'environnement depuis ces soixante dernières années (Sauvé et coll. 2017). En 2015, l'Organisation des Nations unies pour l'éducation, la science et la culture (UNESCO) a proposé une feuille de route pour le secteur de l'éducation à travers la Déclaration d'Incheon et l'agenda Éducation 2030, dans le cadre du Forum Mondial sur l'Éducation (UNESCOa, 2015).

L'agenda Éducation 2030 reconnaît que l'éducation est essentielle pour l'atteinte des objectifs du développement durable et souligne que celle-ci doit être » [...] fondée sur les droits de l'homme et la dignité, la justice sociale, l'inclusion, la protection, la diversité culturelle, linguistique et ethnique, ainsi que sur une responsabilité et une obligation de rendre des comptes partagés » (UNESCO, 2015a, art. 5). En effet, le cadre d'action Éducation 2030 s'accompagne d'un système d'indicateurs établis selon des critères de pertinence, de faisabilité, de communicabilité et d'interprétation. Ces indicateurs permettent de mesurer les progrès en éducation au développement durable pour habiter les territoires selon des perspectives mondiale, régionale, nationale ou thématique (UNESCO, 2015b). Les indicateurs montrent que cette éducation au développement durable devrait s'accompagner de mesures pour la compréhension adéquate des questions socialement vives relatives à la citoyenneté mondiale et à la durabilité. Dans cette perspective, elle sera propice à l'éducation et la formation au Nord comme lieu de pouvoir et de dynamiques spatiales d'humanisation.

\section{Éducation environnementale post-Rio}

Pour contrecarrer des visions de développement jugées écocides, certains auteurs évoquent, dans des publications parfois controversées, des idées pour contribuer au futur de l'éducation à travers le courant post-Rio (post-sustanaibility) ${ }^{8}$. Dans ces mouvances réputées contre-hégémoniques, des représentations du Nord sont mises de l'avant à travers une vision qui s'écarte des structures et des forces sociales pouvant mener l'humanité à l'extinction (Braidotti, 2013; Wiens et coll., 2020). Inspirés des principes de dénuement matériel, certains proposent de se rapprocher de la nature (Mowat, 2009 ; Adock, 2017 ; Jickling et Sterling, 2017), soulignant le dilemme « Need vs Greed $»^{9}$ (Ali, 2009) selon lequel les Peuples du Nord prélèvent de l'environnement le nécessaire à leur survie et à leur reproduction (Need), tandis que les communautés du Sud en tirent plus que le nécessaire pour leur profit (Greed). Ces conceptualisations du Nord invitent à retrouver la nature en nous, au seuil de l'animalité (Cajete, 1994) plutôt que de l'humanité (Loo, 2017). Elles intègrent le sens du lieu, la culture autochtone et les principes de communion avec la nature, favorables à une éducation sur le sens $d u$ Nord comme lieu de vie et lieu de pouvoir. 


\section{Éducation et pédagogie autochtones}

29

Plusieurs auteurs évoquent une pédagogie autochtone précolombienne basée sur des stratégies pour transmettre tout un ensemble de savoirs et de savoir-faire à travers la langue et la culture (Battiste, 2002 ; Blanchet-Cohen, 2017 ; Semin, 2009). Il s'établirait ainsi un équilibre entre l'épanouissement de l'individu et celui du groupe dans l'espace et au-delà du temps (Godlewska, 2013 ; Prévil et Arias-Ortega, 2020). Campeau (2019) propose d'associer la pédagogie autochtone (Battiste, 2002, 2013) à une éducation basée sur le lieu (Barnhardt 2008 ; Somerville et coll., 2011). Cette pédagogie hybride serait de nature à rapprocher des contenus en histoire, écologie et sciences du territoire des légendes et des récits, à travers la langue et la culture, sans négliger la spiritualité ni la perspective autochtone de la science et de la technologie (Campeau, 2019). Forte d'une telle approche, l'éducation au sens du Nord peut contribuer à coconstruire une identité et une vision $\mathrm{du}$ monde, en reliant le développement territorial, la perspective autochtone et les enjeux environnementaux (Barnhardt 2008; Campeau, 2019). L'éducation et la pédagogie autochtones aideront à enseigner le sens du Nord particulièrement comme lieu de vie et lieu de pouvoir, et à enrichir des représentations du Nord comme lieu de dynamiques spatiales d'humanisation.

\section{Exploration de l'interdisciplinarité des approches et des pratiques éducatives}

Devant la complexité des rapports des communautés et des sociétés à l'espace (Steele, 1981 ; Tuan, 2001) émerge la nécessité d'une éducation au sens du Nord comme objet d'apprentissage avec une double finalité éducative: a) la définition du sens des identités territoriales et culturelles Nord/Sud en s'appuyant sur les lieux de vie et les lieux de pouvoir (Bouchard et Désy, 2013) ; b) l'appréhension du sens de la gouvernance des ressources territoriales Sud/Nord en s'appuyant sur les lieux de pouvoir et de dynamiques spatiales d'humanisation (Canobbio, 2009 ; Girault et Barthes, 2016). Les compétences et les référents didactiques essentiels pour favoriser le développement d'identités et la concertation pour la gouvernance des ressources du Nord nécessiteront les capacités d'intégrer le sens du Nord (Audigier, 1997 ; Bonnemaison, 2000 ; Campeau, 2019; Claval, 2012). Cela favorisera l'émergence de percepts (imaginaires), de concepts (données et informations) et d'affects (représentations) pour renforcer l'agentivité comme sentiment de pouvoir agir et s'engager au Nord (Audigier, 2012).

\section{Percepts du Nord}

Les percepts du Nord sont des entités cognitives, constituées d'un ensemble d'informations sélectionnées et structurées selon l'expérience antérieure, et mobilisées dans des contextes expérientiels (Deleuze et Guattari, 2013 ; Déry, 2016). Ils découlent souvent des savoirs locaux ou des valeurs sur le lieu comme milieu de vie et de pouvoir. Ils peuvent être attractifs et positifs (beauté, tranquillité, immensité, diversité, sensibilité ou spiritualité) ou répulsifs (fragilité, hostilité, précarité, animalité, dangerosité ou pauvreté) (Jay-Rayon, 1983). Ils peuvent être revisités, notamment à

Éducation relative à l'environnement, Volume 16-2 | 2021 
travers la géopoétique nordique, les référents post-Rio et les approches de l'éducation et de la pédagogie autochtones.

\section{Concepts du Nord}

32 Les concepts relatifs au Nord découlent de savoirs sur les lieux, et traduisent les observations et les représentations des objets de pensée concrets ou abstraits. Ils permettent de rattacher à ces objets les divers percepts et d'en organiser les connaissances (Deleuze et Guattari, 2013; Jay-Rayon, 1983; Wackermann, 2005). L'enseignement actuel du monde social concernant les enjeux socioterritoriaux circonscrit le territoire-autochtone aux revendications et le territoire-ressource aux finalités énergétiques (Québec, 2010). Ainsi, le territoire-nordique, nouveau concept à définir, pourra s'appuyer sur le développement du sens du Nord. Dans cet esprit, les indicateurs de la nordicité, du développement durable et de l'Éducation 2030 inspireront des contenus prioritaires à apprendre sur le Nord selon les cycles et les domaines de formation.

\section{Affects pour le Nord}

Les affects pour le Nord sont liés à l'agentivité du Nord. Ils se manifestent par des attitudes et des habiletés exercées ou éprouvées, et témoignent d'une compétence sur une entité et ses relations (percept ou concept) par la pratique et l'intellect (Deleuze et Guattari, 2013; Jay-Rayon, 1983; Sauvé, 2014). Les groupes humains ont appris à intégrer tout un ensemble de savoirs, de savoir-faire et de savoir-être encodés dans la langue et la culture. Par l'apprentissage social, la transmission culturelle et l'accompagnement, il est possible d'acquérir des fondamentaux éducatifs expérientiels. Également, le développement du pouvoir d'agir peut s'enrichir des savoirs découlant de l'éducation et de la pédagogie autochtones pour donner du sens au Nord comme lieu de vie et lieu de pouvoir (Kudryavtsev et coll., 2012).

Le sens du Nord comme objet d'apprentissage s'accompagne d'une démarche pour « faire vivre et connaître " le Nord grâce au développement d'un sens du lieu par les imaginaires (percepts), les données et les modèles (concepts), et les représentations (affects) propices à l'agentivité comme sentiment du pouvoir d'agir. Pour ce faire, on devra recourir à des processus d'investigation des réalités socioterritoriales et à l'enseignement des spécificités locales (Godlewska, 2013 ; Prévil et Arias-Ortega, 2020). Les affects liés au sens $d u$ Nord aideront à développer des compétences pour entreprendre des projets collectifs visant à résoudre des problèmes sociaux et communautaires, et permettre à des groupes culturels spécifiques et/ou minoritaires de prendre du pouvoir sur leurs lieux de vie. La collaboration avec les communautés autochtones ou locales, qui maîtrisent des savoirs multiples relativement au lieu, pourra être organisée à travers les laboratoires-terrains et l'enrichissement des contenus éducatifs. Cette mise en œuvre ne devra pas s'appuyer uniquement sur des " dires d'experts » : elle nécessitera la co-construction des savoirs. Ainsi, l'éducation au sens $d u$ Nord pourra prétendre à un caractère essentiel pour l'éducation au Québec et au Canada (Battiste, 2013 ; Hamelin, 2006). 


\section{Conclusion}

Le système éducatif doit se renouveler au rythme des transformations sociales, pour préparer les apprenants à affronter la complexité des enjeux et des réalités du $21^{\mathrm{e}}$ siècle (UNESCO, 2015a). Cet article traite de l'opportunité d'un renouvellement de l'éducation géographique en vue d'y intégrer le sens du Nord comme modalité du sens du lieu (sense of place) dans la formation au monde social. Ce renouvellement implique le développement de savoirs sur les espaces et les territoires (Mérenne-Shoumaker, 2019), et une réactualisation des façons de coconstruire des attitudes et des comportements personnels et sociaux vis-à-vis des systèmes socioécologiques pour prendre position, s'engager et savoir agir (Audigier, 2015). Les compétences à développer seront utiles pour protéger ces systèmes socioécologiques et les améliorer, ou corriger leurs fractures ou leurs dysfonctionnements (Lengen et Kistemann, 2012).

Nous avons caractérisé le sens du Nord comme nouvel objet d'apprentissage et exploré six approches pouvant contribuer au renouvellement de l'éducation géographique. Ce nouvel objet d'apprentissage pourra avoir un impact particulier en permettant aux apprenants de développer un affect du Nord, avec ses lieux de vie, de pouvoir et de dynamiques spatiales d'humanisation. C'est ainsi que l'éducation géographique pourra contribuer à développer l'agentivité et à former un individu épanoui, un citoyen engagé et un travailleur responsable (Québec, 2010).

Les enjeux de l'éducation au sens du Nord soulèvent la nécessité de développer de nouveaux repères pour la transmission de savoirs-clés et l'émergence de nouvelles compétences sur le sens du lieu (sense of place) au Nord (Hamelin, 2006 ; Harvey, 1994). Ces savoirs et ces compétences seront les garants de la co-construction d'identités territoriales et culturelles, et de l'appréhension des capacités de négociation territoriale favorables au renforcement de l'écocitoyenneté. L'éducation au sens $d u$ Nord s'inscrit ainsi dans une démarche éducative capable d'intégrer des préoccupations critiques, éthiques et politiques (Sauvé, 2014) dans les processus d'investigation des réalités environnementales, sociales et culturelles problématiques au Québec et au Canada.

\section{BIBLIOGRAPHIE}

Ali, S. (2009). Treasures of the earth : need, greed, and a sustainable future. New Haven : Yale University Press.

Allison Mitcham, (1983). The Northern Imagination. A Study of Canadian Literature. Moonbeam : Penumba Press.

Anderson, B. (2006). Imagined Communities : Reflections on the Origin and Spread of Nationalism. New York : Verso Books.

Arendt, H. (1972). La crise de la culture-Huit exercices de pensée politique. Paris : Gallimard. 
Asselin, H. (2011). Plan Nord : les Autochtones laissés en plan. Recherches amérindiennes au Québec, 41(1), 37-46.

Audigier, F. (1997). Histoire et géographie : Un modèle disciplinaire pour penser l'identité professionnelle. Recherche \& formation, 25(1), 9-21.

Audigier, F. (2012). Les Éducation à... et la formation au monde social. Recherches en didactiques, $14(2), 47-63$.

Audigier, F. (2015). Éducation à... et préparation à la vie. Dans F. Audigier, A. Sgard et N. TutiauxGuillon (Dir.), Sciences de la nature et de la société dans une école en mutation : Fragmentations, recompositions, nouvelles alliances? (p. 25-35). Louvain-la-Neuve : De Boeck Supérieur.

Barnhardt, R. (2008). Creating a place for Indigenous Knowledge in Education. Dans D. Gruenewald \& G. Smith (Eds.), Place-based Education in the Global age : Local Diversity (p. 113-133). New York : Taylor \& Francis Group.

Battiste, M. (2002). Indigenous Knowledge and Pedagogy in First Nations Education. A Literature Review. Ottawa : Indian and Northern Affairs Canada. Consulté sur http://tinyurl.com/llayfcm.

Battiste, M. (2013). Decolonizing education : Nourishing the learning spirit. Saskatoon : Purich Publishing.

Bayly, J. (2018). Nord. Ottawa : Encyclopédie Canadienne. Consulté sur https:// www.thecanadianencyclopedia.ca/fr/article/nord-5

Benimmas, A. et Blain, S. (2019). Créer une carte narrative sur Google Maps : étude du raisonnement géographique et de la cohérence textuelle chez les élèves de la $9^{\mathrm{e}}$ année secondaire. Éducation et francophonie, 47(2), 145-171.

Bocking, S. et Martin, B. (2017). Ice Blink : Navigating Northern Environmental History. Calgary : University of Calgary Press.

Bone, R. (2016). The Canadian North : Issues and Challenges. Don Mills : Oxford University Press. Bonnemaison, J. (2000). La géographie culturelle. Paris : Éditions du CTHS.

Bouchard, S. et Désy, J. (2013). L’Objectif Nord : le Québec au-delà du 49e. Québec : Sylvain Harvey. Bouvet, R. (2015). Vers une approche géopoétique : lectures de Kenneth White, de Victor Segalen et de J.MG Le Clézio. Québec : Presses de l'Université du Québec.

Braidotti, R. (2013). Posthuman Humanities. European Educational Research Journal 12(1), 1-19.

Campeau, D. (2019). Pédagogie autochtone et pédagogie du lieu : démarche hybride pour l'intégration de dimensions culturelles autochtones dans l'enseignement au primaire au Québec. Thèse de doctorat inédite : Université de Sherbrooke. Consulté sur https://savoirs.usherbrooke.ca/handle/ $11143 / 15042$

Canada (2009). Stratégie pour le Nord du Canada : notre Nord, notre patrimoine, notre avenir. Ottawa : Gouvernement du Canada.

Cannatella, H. (2007). Place and being. Educational Philosophy and Theory, 39(6), 622- 632.

Canobbio, É. (2009). Géopolitique d'une ambition Inuite : le Québec face à son destin nordique. Québec : Septentrion.

Chartier, D. (2014). La nordicité du Québec : Entretiens avec Louis-Edmond Hamelin. Montréal : Presses de l'Université du Québec. 
Cheng, A., Kruger, L. E. et Daniels, S.E. (2003). "Place" As an Integrating Concept in Natural Resource Politics : Propositions for a Social Science Research Agenda. Society \& Natural Resources : An International Journal, 16(2), 87-104.

Claval, P. (2012). Géographie culturelle : une nouvelle approche des sociétés et des milieux. Paris : Armand Colin.

Colin, B. (2013). The Geography of Education and Comparative Education. Comparative Education, $49(3), 275-289$

Commission de vérité et réconciliation du Canada (CRV) (2015). Honorer la vérité, réconcilier pour l'avenir. Sommaire du rapport final de la Commission de vérité et réconciliation du Canada. Montréal/ Kingston/London/Chicago/McGill : Queen's University Press.

Cossio, R. (2015). Teorías de la cultura y diagnóstico sobre la educación intercultural en el Perú en sus aspectos lingüísticos y literarios (Doctoral dissertation). Universidad de Granada : Espana.

Cottereau, D. (1999). Chemins de l'imaginaire, Pédagogie de l'imaginaire en éducation à l'environnement. Paris : Éditions de Babio.

Deleuze, G. et Guattari, F. (2013). Qu'est-ce que la philosophie ? Paris : Minuit.

Déry, C. (2016). Enseigner les enjeux du Nord québécois et la nordicité en géographie ; quelques pistes pour le $3^{\mathrm{e}}$ cycle du primaire et le $1^{\mathrm{er}}$ cycle du secondaire. Enjeux de l'univers social 12 (3), 13-15.

Desbiens, C. (2012). 10 Idées pour le Nord : un manifeste pour la nordicité. Cahiers de géographie du Québec, 56(159), 643-659.

Société du Plan Nord (2019). Documents du Plan Nord. Consulté sur https://plannord.gouv.qc.ca/ $\mathrm{fr} / \mathrm{spn} /$ documentation/.

Feit, H. (2018). Dispossession with Possession, Governance with Colonialism : Algonquian Hunting Territories and Anthropology as Engaged Practice. Anthropologica 60, 149-160. Consulté sur http:// hdl.handle.net/11375/23928.

François, L (dir.) (2008). Intelligence territoriale. Paris : Lavoisier.

Girard, C., \& Brisson, C. (2018). Reconnaissance et exclusion des peuples autochtones au Québec : du traité d'Alliance de 1603 à nos jours. Laval : Presses de l'Université Laval.

Girault, Y. et Barthes, A. (2016). Postures épistémologiques et cadres théoriques des principaux courants de l'éducation aux territoires. Éducation Relative à l'Environnement 13(2). Consulté sur https://journals.openedition.org/ere/755

Godlewska, A. (2013). A deeper sense of place : Awakening to belonging. Dans J. T. Johnson \& S. C. Larsen (Eds.), A deeper sense of place : Stories \& journeys of Indigenous-academic collaboration ( $p$. 217-231). Corvallis : Oregon State University Press, 217-231.

Grace, S. (2007). Canada and the Idea of North. Montreal : McGill.

Griffiths, F., Huebert, R. N. et Lackenbauer, P. W. (2015). Le Canada et l'Arctique. Montréal : Presses de l'Université de Montréal.

Hamelin, L. E. (1976). Nordicité canadienne. Québec : Hurtubise, collection « Cahiers du Québec » Hamelin, L. E. (2006). L'âme de la terre: Parcours d'un géographe. Québec : Éd. MultiMondes.

Harvey, F. (1994). L'historiographie du Nord du Québec. Recherches sociographiques, 35(3), 373-420. Consulté sur https://www.erudit.org/fr/revues/rs/1994-v35-n3-rs1592/056898ar/ 
Hulan, R. (2014). Northern Experience and the Myths of Canadian Culture. Montréal : McGill-Queen's University Press.

Jay-Rayon J. (1983). Pour prendre le temps d'être mieux. Sillery : Québec science.

Jickling, B., et Sterling, S. (Eds.). (2017). Post-sustainability and environmental education : Remaking education for the future. Thunder Bay : Springer.

Kudryavtsev, A., Stedman, R. et Krasny, M. (2012). Sense of place in environmental education. Environmental Education Research, 18(2), 229-250.

Lengen, C., et Kistemann, T. (2012). Sense of Place and Place Identity : Review of Neuroscientific Evidence, Health \& Place 18, 1162-1171.

Mérenne-Schoumaker, B. (2017). Didactique de la géographie : organiser les apprentissages. Bruxelles : De Boeck.

Mérenne-Schoumaker, B. (2019). Apports et finalité de la géographie dans une formation de base. Réflexions et propositions. Éducation et francophonie, 47(2), 8-23.

Mialaret, G. (2006). Sciences de l'éducation : aspects historiques, problèmes épistémologiques. Paris : Presses universitaire de France.

Mowat, F. ([1963] 2009). Never cry wolf. Toronto : McClelland \& Stewart.

Nations Unies. (1992). Déclaration de Rio sur l'environnement et le développement. Rio de Janeiro : Assemblée Générale A/CONF.

Niens, U. et Reilly, J. (2012). Education for Global Citizenship in a Divided Society ? Young People's Views and Experiences. Comparative Education, 48(1), 103-118.

Nungak, Z. (2017). Contre le colonialisme dopé aux stéroïdes. Montréal : Boréal.

Nungak, Z. (2019). Contre le colonialisme dopé aux stéroïdes : le combat des Inuits du Québec pour leurs terres ancestrales. Montréal : Boréal.

Ottum, L et Seth T. (2016). Wordsworth and the Green Romantics : Affect and Ecology in the Nineteenth Century. Durham : University of New Hampshire Press. Consulté sur muse.jhu.edu/book/44867.

Ouranos (2015). Vers l'adaptation. Synthèse des connaissances sur les changements climatiques au Québec. Partie 1 : Évolution climatique au Québec. Montréal : Ouranos.

Pélissier, P. (1989). Pourquoi enseigner la géographie ? L'Espace Géographique, 18(2), 185-185.

Consulté sur www.jstor.org/stable/44382398

Peters, F., (2015). Systèmes scolaires. Encyclopédie Canadienne. Consulté sur https:// www.thecanadianencyclopedia.ca/fr/article/systemes-scolaires

Pichon, M. (2015). Espace vécu, perceptions, cartes mentales : l'émergence d'un intérêt pour les représentations symboliques dans la géographie française (1966-1985). Bulletin de l'association de géographes français, 92, 95-110.

Prévil C., et Arias-Ortega, K. (2020). Persistance de la crise éducative autochtone au Québec (Canada) et dans l'Araucania (Chili), une approche comparative (soumis). Dans Maheux, G., Quintriqueo, S., Bacon, L. et Pellerin, G., La décolonisation de la scolarisation en milieu autochtone : exploration de différentes approches (p. 15-44). Québec : Presses de l'Université du Québec, 15-44.

Prévil, C. (2009). Participation du public dans la gouvernance de l'environnement et $d u$ territoire : pour améliorer l'instrumentation. [Vertigo] La revue électronique en sciences de l'environnement, 9(1). Consulté sur https://journals.openedition.org/vertigo/8580 
Pumain, D. et Saint-Julien, T. (1997). L'analyse spatiale. Localisation dans l'espace. Paris : Armand Colin.

Québec (2006). Loi sur le développement durable. Projet de loi (118). Québec : Gouvernement du Québec.

Québec. (2010). Programme de formation de l'école québécoise. Éducation préscolaire et enseignement primaire. Ministère de l'éducation : Gouvernement du Québec 2001-2010. Consulté sur http:// www.education.gouv.qc.ca/enseignants/pfeq/

Québec. (2012) : Document synthèse sur les indicateurs québécois de développement durable. Documentation gouvernementale : Gouvernement du Québec.

Rougier, H. (1991). Le Nord canadien est-il un défi à la géographie? Cahiers franco-canadiens de l'ouest 3(2), 217-232.

Sauvé, L. (2014). Au cœur des questions socio-écologiques : des savoirs à construire, des compétences à développer. Éducation relative à l'environnement, 11. Consulté sur https:// journals.openedition.org/ere/662

Sauvé, L., Orellana, I., Villemagne, C. et Bader, B. (2017). Éducation, environnement, écocitoyenneté : repères contemporains. Québec : Presses de l'Université du Québec

Scannell, L., et Gifford, R. (2010). Defining Place Attachment : A Tripartite Organizing Framework. Journal of environmental psychology, 30(1), 1-10.

Scheibling, J. (2011). Qu'est-ce-que la géographie ? Paris : Hachette Éducation.

Colin, S. (2013). Le partage des ressources au Québec : perspectives et stratégies autochtones. Dans A. Beaulieu, S. Gervais et M. Papillon (eds), Les Autochtones et le Québec : Des premiers contacts au Plan Nord (p. 363-384). Montréal : Presses de l'Université de Montréal.

Semin, G. (2009). Language, Culture, Cognition. How Do They Intersect ? Problems and Solutions. Dans Robert S. Wyer, Chi-yue Chiu, Ying-yi Hong, Understanding Culture : Theory, Research and Application. New York : Psychology Press.

Somerville, M., Davies, B., Power, K., Gannon, S. et Carteret, P. (2011). Place pedagogy change. Rotterdam : Sense Publishers.

StatCan (Statistique Canada) (2017). Les peuples autochtones au Canada : faits saillants du Recensement de 2016. Consulté sur https://www150.statcan.gc.ca/n1/daily-quotidien/171025/dq171025afra.pdf

Steele, F. (1981). The Sense of Place. Boston : CBI Publishing Company.

Tuan, Yi-Fu. (1977/2001). Space and Place: The Perspective of Experience. Minneapolis : University of Minnesota Press.

UNESCO (2015a). Éducation 2030, Déclaration d'Incheon et Cadre d'action pour la mise en œuvre de l'objectif de développement durable. UNESCO : Document de programme et de réunion. ED-2016/WS/ 28 4. Consulté sur https://unesdoc.unesco.org/ark :/48223/pf0000245656_fre

UNESCO, (2015b) : Indicateurs thématiques pour le suivi de l'agenda de l'Éducation 2030. Proposition du Groupe consultatif technique. Consulté sur http://uis.unesco.org/sites/default/files/documents/ thematic-indicators-to-monitor-the-education-2030-agenda-technical-advisory-groupproposal-2015-fr.pdf 
University of Chicago Press Journals. (2020). Goodbye 'extinction,' hello 'evanescence' ? Validating a new paradigm. ScienceDaily. Consulté sur www.sciencedaily.com/releases/ 2020/06/200618120159.htm

Vaillancourt, M. (2017). Un héritage à habiter : Lecture géopoétique de Kuessipan/À toi et de Puamun, le rêve, de Naomi Fontaine. Recherches amérindiennes au Québec, 47(1), 25-34.

Wackermann, G. (Coord) (2005). Dictionnaire de géographie. Paris : Ellipses.

Wiens, D., Sweet, T. et Worsley, T. (2020). Validating the New Paradigm for Extinction : Overcoming 200 Years of Historical Neglect, Philosophical Misconception, and Inadequate Language. The Quarterly Review of Biology, 95(2), 109-124.

\section{NOTES}

1. Une ébauche de ce texte a fait l'objet d'un article soumis dans les Actes de la $21^{e}$ édition du Congrès d'études inuit.

2. Le Conseil de l'Arctique est formé de huit États membres (Canada, Danemark, ÉtatsUnis, Finlande, Islande, Norvège, Russie, et Suède), six organisations internationales permanentes d'Autochtones de l'Arctique, quatorze États observateurs et une vingtaine d'organisations gouvernementales ou non gouvernementales: https://arcticcouncil.org/en/

3. Au Québec, le programme de géographie au secondaire privilégie le développement de trois compétences à travers quatre enjeux territoriaux et cinq territoires-types (Québec, 2010).

4. Le Nouveau Brunswick et la Nouvelle-Écosse ne détiennent pas de territoire au Nord comme défini dans le texte.

5. Débats sociaux sur le Nord au Québec depuis 2008 : Forum Plan Nord (2012) : Citoyens CSN, CSQ, Chaire de Recherche du Canada, Femmes Autochtones, Nature Québec, etc. ; Objectif Nord (2013) : Télé-Québec; Dossier Nord (2011) : Le Devoir; La Presse; Le Journal; Radio Canada ; Association d'Entreprises Minières et Blogs ; etc.

6. Principe de l'Until Policy: «en territoire réclamé, le développement économique des non-Autochtones ne peut arriver qu'après une solution aux revendications foncières [...]» (Hamelin, 2006, p. 96).

7. Portant sur l'environnement et la consommation, la diversité, le vivre-ensemble et la citoyenneté (Québec, 2010).

8. En référence à l'après Somment de la Terre (Sommet de Rio), 1992.

9. Ali (2009) écrit: «Mahatma Gandhi once famously said that 'the earth has enough resources for our need but not for our greed'« . 


\section{RÉSUMÉS}

Les études sur la nordicité ont pris un essor considérable dans l'ensemble des agendas fédéraux et provinciaux depuis le tournant du millénaire au Canada. Toutefois, il est permis de se questionner sur le retard de l'éducation et de la formation en géographie au Québec, à s'intéresser au sens $d u$ Nord. Historiquement, l'éducation formelle a très peu considéré en effet les connaissances issues des communautés nordiques. Cet article examine la conceptualisation de nouveaux savoirs sur le sens du Nord comme sens du lieu (sense of place) au Québec et au Canada. L'élaboration de savoirs essentiels à enseigner et de compétences à développer sur le Nord peut constituer une réelle opportunité pour le renouvellement de l'éducation géographique et de l'éducation relative à l'environnement. Un tel renouvellement pourra contribuer à promouvoir l'écocitoyenneté dans la perspective du développement d'identités territoriales et culturelles, et de la prise en compte de nouvelles dynamiques spatiales d'humanisation.

Northern studies have grown considerably across Canada since the last few decades. However, it appears a persistent delay in the education and training to the sense of the North, for a comprehensive teaching of Geography in Québec. Historically, the geographic education seems reluctant for including contextual knowledge coming from the Northern communities in placebased education. This paper discusses the opportunity of conceptualizing new knowledge on sense of North as Sense of Place in Québec and Canada. Elaboration of new capacities and essential knowledge about the North could constitute a thrilling opportunity for the renewal of the geographic education and environmental education. This renewed geographic education will help enhancing eco-citizenship by including northern perspectives in territorial and cultural identities as well as new spatial dynamics of humanization.

\section{INDEX}

Keywords : sense of place, geographic education, place based education, interculturalism, ecocitizenship

Mots-clés : sens du lieu, éducation géographique, éducation au lieu, interculturalisme, écocitoyenneté

\section{AUTEURS}

\section{CARLO PRÉVIL}

Professeur à l'Université du Québec en Abitibi Témiscamingue et chercheur associé au Centr'ERE, ses intérêts de recherche portent sur l'application des méthodes de la science de l'information géographique et de l'analyse multicritère dans l'aménagement du territoire. Il s'attarde particulièrement à la caractérisation du sens du lieu, à l'éducation relative à l'environnement, à l'éducation comparée, à la modélisation territoriale et à l'implémentation d'instruments de connaissances pour faciliter la participation du public dans la concertation et l'aide à la décision territoriale.

\section{KATERIN ARIAS-ORTEGA}

Professeure à l'Université Catholique de Temuco (UCT, Chili), ses principales contributions ont porté jusqu'ici sur différents champs de l'éducation, notamment : les fondements et 
l'épistémologie de l'éducation, le développement de curriculum et l'évaluation, l'administration scolaire, l'éducation interculturelle, l'éducation autochtone et l'éducation comparée. Ses plus récents travaux portent sur les enjeux de l'accompagnement, de la coopération et de la communication dans les pratiques professionnelles des agents éducatifs impliqués dans l'éducation interculturelle. 